\title{
L’oeuvre des Missionnaires Oblates du Sacré-Coeur et de Marie Immaculée dans l'Église
}

\section{Cécile d'Amours}

Volume 37, 1970

La vie religieuse au Manitoba

URI : https://id.erudit.org/iderudit/1007286ar

DOI : https://doi.org/10.7202/1007286ar

Aller au sommaire du numéro

Éditeur(s)

Les Éditions Historia Ecclesiæ Catholicæ Canadensis Inc.

ISSN

0318-6172 (imprimé)

1927-7067 (numérique)

Découvrir la revue

Citer cet article

d'Amours, C. (1970). L’oeuvre des Missionnaires Oblates du Sacré-Coeur et de Marie Immaculée dans l'Église. Sessions d'étude - Société canadienne d'histoire de l'Église catholique, 37, 247-258. https://doi.org/10.7202/1007286ar

Tous droits réservés @ Les Éditions Historia Ecclesiæ Catholicæ Canadensis Inc., 1972
Ce document est protégé par la loi sur le droit d'auteur. L'utilisation des services d'Érudit (y compris la reproduction) est assujettie à sa politique d'utilisation que vous pouvez consulter en ligne.

https://apropos.erudit.org/fr/usagers/politique-dutilisation/ 


\section{L'œuvre des Missionnaires Oblates du Sacré-Cœur et de Marie Immaculée dans l'Église}

La communauté des Sœurs Missionnaires Oblates du Sacré-Cœur et de Marie Immaculée, par son esprit et son but, se rattache aux Provencher et aux Taché: donner aux enfants un enseignement chrétien et préparer des générations utiles à l'Église et au pays.

Comme partout ailleurs, l'Église s'est faite, dans l'Ouest, fondatrice d'écoles. Dès l'année qui suit leur arrivée à la Rivière Rouge, en 1818, les missionnaires que furent monseigneur Provencher et l'abbé Dumoulin ouvrent deux écoles. Jusqu'en 1870 les habitants de la Rivière Rouge jouissent, pour l'éducation de leurs enfants, d'un véritable régime de liberté. Catholiques et protestants ont leurs écoles séparées et contribuent respectivement à leur soutien.

En 1870, lors de l'entrée du Manitoba dans la Confédération, les Canadiens français de la province, ay ant à leur tête monseigneur Taché, exigeaient le maintien des écoles séparées et l'usage des deux langues, française et anglaise, dans la Législature et dans les cours. L'Acte du Manitoba garantissait ces droits.

Vingt ans après l'entrée du Manitoba dans la Confédération, les législateurs n'hésitent pas à fouler aux pieds les promesses solennelles faites au peuple de la Rivière Rouge par le représentant de la couronne britannique. Le Manitoba devenait la première province séparatiste.

Vers 1900, Saint-Boniface justifiait assez bien le nom de « Cathedral City " dont l'avaient gratifiée les citoyens de Winnipeg. Saint-Boniface est l'église-mère de la catholicité dans tout l'Ouest canadien. Elle a subi plusieurs morcellements: la Colombie (Vancouver, archidiocèse) 1908; l'Alberta (Edmonton, archidiocèse) 1912; la Saskatchewan (Regina, archidiocèse) 1915 et, surtout, en 1915 aussi, la création, pour le moins prématurée, de l'archidiocèse de Winnipeg.

Des Institutions assez nombreuses, œuvres d'enseignement, œuvres de charité, y florissaient sous la houlette d'un pasteur intrépide autant que zélé, et faisaient de la ville épiscopale un foyer de vie catholique intense, vie dont le mouvement semblait quelque peu paralysé par une cause profonde dont les effets se faisaient sentir depuis près de quinze ans. 
La trop célèbre et douloureuse question des écoles du Manitoba, qui avait hâté la mort de monseigneur Taché, venait de passer par une de ces crises les plus épineuses et, de l'issue des négociations entreprises, on le croyait du moins, dépendait l'avenir de la race française et de la foi catholique dans les pays nouveaux qui s'ouvraient à la colonisation et à l'immigration.

A monseigneur Taché avait succédé, en 1895, monseigneur LouisPhilippe-Adélard Langevin, oblat de Marie Immaculée, qui devait auréoler d'une gloire non moins pure le siège archiépiscopal de SaintBoniface. La présente année marque le $75^{\circ}$ anniversaire de sa consécration épiscopale. Il avait reçu des mains de son prédécesseur un héritage dont la libre possession paraissait menacée de toutes parts et, dans son ardeur pour sauvegarder les âmes confiées à sa charge, il avait pris comme devise la recommandation de saint Paul à Timothée: "Garde le dépôt ".

Ce dépôt de la foi et de la langue de ses ouailles, monseigneur Langevin avait été bien préparé pour en être le fidèle gardien. Né luimême d'une de ces anciennes familles de la province de Québec établie à Saint-Isidore de Laprairie, il tenait d'elle sa foi robuste, son amour du sol et des traditions de la patrie canadienne.

Depuis sa consécration épiscopale, le 19 mars 1895, avec les députés canadiens-français et tous les hommes bien-pensants, il s'était efforcé, mais sans beaucoup de succès, de rentrer en possession des droits enlevés à la population catholique par la loi de 1890 .

Chaque époque, comme chaque pays d'ailleurs, a ses besoins spéciaux et c'est précisément de la lutte faite contre l'école catholique au pays manitobain qu'apparut à monseigneur Langevin la nécessité d'une congrégation nouvelle de religieuses institutrices pour travailler, avec les instituts déjà établis, à défendre l'enfance menacée dans sa foi et dans sa langue.

Durant une accalmie, les députés catholiques à la Chambre avaient obtenu, du Bureau d'Éducation, que des cours d'École normale soient donnés à l'hiver de 1899 , sous la direction d'un inspecteur canadienfrançais et d'un inspecteur de langue anglaise.

Ces cours eurent d'abord l'aspect d'une école ambulante: une partie des leçons se donnaient à l'ancien Collège de Saint-Boniface, dans un parloir aménagé à cet effet; l'autre, au couvent des Sœurs des Saints Noms de Jésus et de Marie; ou au deuxième étage du magasin Leclerc, ou ailleurs.

Sur de nouvelles instances de l'archevêque et des catholiques éminents qui le soutenaient, on obtint enfin du Gouvernement, en 1902, la construction de l'École normale, angle des rues Masson et Aulneau. 
Le terrain appartenait à la Corporation archiépiscopale de SaintBoniface; il fut donné à condition que le Gouvernement y construisît la maison.

Mais une nouvelle vague de fanatisme était toujours à redouter. Dans ces conditions, le métropolitain de l'Ouest canadien crut que le meilleur moyen de conserver les bribes des droits reconnus par la loi de 1896 aux Canadiens français catholiques - et de préserver la foi dans l'âme des enfants des autres nationalités du diocèse - serait de confier à des religieuses le plus grand nombre possible d'écoles.

A cette époque, très peu de laïcs se donnaient à l'enseignement.

Où trouver des religieuses en nombre suffisant? Les congrégations existantes, qui faisaient un travail admirable et fructueux dans leurs écoles, ne pouvaient suffire à la täche, ni obtenir de renfort de leur maison mère; les autres congrégations du Québec refusaient, faute de sujets, de faire des fondations dans l'Ouest; celles qui venaient de l'étranger, poussées elles aussi sur nos rivages par la persécution religieuse, ne possédaient pas les brevets du pays; ... alors l'archevêque de Saint-Boniface résolut de fonder une NOUVELLE congrégation. Voici comment elle prit racine.

Pour faire suite à son projet, monseigneur Langevin fit construire, en même temps que l'École normale, sur un lot voisin, une maison qui devait recevoir les premières recrues de l'œuvre dont il sentait de plus en plus la nécessité.

Dès le mois de décembre 1902, la maison abritait quatre personnes. La pièce principale fut immédiatement préparée pour recevoir le SaintSacrement qui devait y demeurer en permanence. De là, le titre de Maison-Chapelle, maison-chapelle qui, en fait, était la maison de pension pour les futures institutrices. Quelques dames pensionnaires y seraient reçues. Une douzaine de collégiens, protégés de la Corporation épiscopale, y prendraient leurs repas.

Il n'était cependant pas dans les desseins de Dieu, ni dans les vues de son fondateur, que l'œuvre restât dans ces conditions. Bientôt il fut question d'ériger en communauté religieuse le personnel de la maison. Par suite de certaines difficultés, la chose s'avéra impossible.

Un autre essai d'organisation fut tenté à l'été de 1903 et n'obtint, comme le premier, aucun succès. Ce ne fut qu'au mois de mars 1904 que la réalisation de ce projet devint possible par l'arrivée de quelques nouvelles recrues au nombre desquelles se trouvaient mesdemoiselles Alma Laurendeau et Ida Lafricain. Elles devaient être les pierres d'assise de la congrégation naissante. La généreuse coopération avec laquelle elles entrèrent dans les vues du fondateur leur mérite, à juste titre, le nom de cofondatrices. 
Mademoiselle Alma Laurendeau, devenue plus tard Mère MarieJoseph-du-Sacré-Cœur, était née à Saint-Cyrille de l'Islet, Qué., le 5 novembre 1879. Son père, Louis Laurendeau, originaire de SaintCyrille, était cultivateur. En 1880, il vint à Saint-Boniface. Il fit l'acquisition d'un lot de terre, sur l'une des rues qui devint l'avenue Provencher. Cette propriété appartient encore à la plus jeune de ses filles, madame Gracia Fortin.

La mère de mademoiselle Laurendeau, née Augustine Lebourdais, était la fille de Joseph Lebourdais, pilote, de l'Islet, et d'Henriette Bernier, apparentée à tous les Bernier et Kéroack qui sont venus s'établir au Manitoba.

Par goût plus que par nécessité, elle fait trois ans d'enseignement. Elle s'engage dans les liens du mariage, sans attirance marquée, à tel point que l'on peut se demander si la Providence n'a pas permis le sacrifice d'un attrait inconscient pour une autre vocation afin de mieux seconder les desseins de Dieu.

Mademoiselle Lebourdais n'avait pas connu de religieuse dans sa jeunesse. Aussi, tout en laissant à ses cinq filles une grande liberté dans leurs décisions, elle fut heureuse de compter trois religieuses parmi elles. Elle leur communiqua, sans doute, ses goûts d'institutrice: quatre ont consacré un total d'une centaine d'années au service de la jeunesse catholique manitobaine. Alma, l'aînée des filles, fit ses études à l'Académie Taché, devenue Hôpital Taché, sous la direction des Sours Grises de la Charité. Elle enseigna six ans avant d'entrer à la MaisonChapelle.

Mademoiselle Lafricain, devenue plus tard Mère Saint-Viateur, naquit à Montréal, le 2 février 1871. Elle était la première enfant d'Henri Lafricain, originaire de Montréal, et d'Hedwige Laporte, née à Plattsburg, New York.

Ses études terminées, mademoiselle Lafricain demeura dans sa famille jusqu'à l'âge de 21 ans, puis se retira, le $1^{\text {er }}$ mai 1894, chez une dame Poitou qui avait ouvert une maison d'œuvres portant le nom "Béthanie ». Quelques personnes, sous la conduite du père Almire Pichon, s.j., l'aidaient à visiter les familles pauvres, à donner l'instruction catéchistique aux enfants arriérés ou abandonnés, etc.

Dans cette maison, mademoiselle Ida Lafricain rencontra mademoiselle Délia Tétrault, la future fondatrice de la Société des Sours Missionnaires de l'Immaculée Conception. Madame Poitou était veuve et n'avait nullement en vue la fondation d'un institut religieux, alors que mesdemoiselles Tétrault et Lafricain songeaient à se consacrer à Dieu dans l'état religieux. 
Après huit ans passés dans cette maison, elles la quittèrent avec l'intention d'entreprendre la fondation d'une communauté religieuse.

Lors d'un voyage à Montréal, à l'automne 1903, monseigneur Langevin eut l'occasion de visiter l'École Apostolique où mademoiselle Tétrault, avec ses compagnes, attendait l'heure de Dieu pour réaliser l'œuvre projetée.

Monseigneur crut que mademoiselle Lafricain était un sujet possible et désirable pour l'établissement de sa communauté. Il l'invita à venir au Manitoba. La première réaction fut un moment de surprise, mais la grâce et la prière obtinrent ce que la nature et les liens noués avec cette maison de Montréal n'auraient pu laisser prévoir.

La Providence semblait avoir fait croiser le chemin de ces deux fenumes pour leur apprendre le détachement et le sacrifice qui devaient féconder leur mission respective.

Mademoiselle Ida Lafricain arrive à Saint-Boniface le 19 mars 1904. Elle accepte de se joindre aux aspirantes déjà en retraite préparatoire à la cérémonie de vêture qui eut lieu cinq jours plus tard. Cette cérémonie de vêture ne fut pas un événement retentissant. Les circonstances de lieu se prêtaient mal à de grandes démonstrations. La chapelle était petite. En fait d'invités... quelques prêtres et les proches parents des nouvelles novices. L'espace manquait... même pour placer une table sur laquelle seraient déposés les habits; on prit le parti le plus simple: un banc où se trouvaient les prie-Dieu pour la communion.

Afin de mieux faire connaître le but de la congrégation et les motifs du fondateur, à la suite de l'interrogatoire, monsieur l'abbé Joseph Trudel, secrétaire, lut le document d'érection canonique du noviciat, et la cérémonie reprit son cours. Elle se termina par la Bénédiction du Saint-Sacrement.

A ce moment, on put voir les nouvelles novices reprendre une partie de leurs fonctions: la sacristine allume les cierges, l'organiste et les deux chanteuses se rendent à leur poste. Pour un instant, il en reste deux au pied de l'autel. Comme au temps de monseigneur Taché où tout le personnel de l'évêché était évêque ... chez nous, tout le personnel était novice. Et les novices devaient, même en un jour de prise d'habit, remplir leurs fonctions ordinaires.

Ce n'en était pas moins la naissance dans l'Église d'un nouvel organisme appelé à jouer son rôle dans le pays de son origine. Longtemps, la semence jetée dans le sillon par le semeur qu'était monseigneur Langevin avait pu dormir, puis mourir, pour enfin renaître. La lutte, néanmoins, n'était pas finie. Le jeune institut, frêle arbrisseau, allait subir maintenant les assauts de la tempête que son apparition devait 
susciter. Au dehors et au-dedans, il sera secoué par des vents contraires. Ses racines, lentement enfoncées dans le sol manitobain, le rendront capable de résister à tous les orages.

Monseigneur s'occupa activement de donner à la nouvelle congrégation les premières Règles qui devaient la constituer en un corps religieux. Quatre jours après, les jeunes novices recevaient un règlement d'essais et de pressantes exhortations à en bien observer tous les points.

Depuis le 24 mars, de nombreuses visites de monseigneur avaient permis à la petite famille d'apprécier encore davantage sa grande bonté et son vif désir de la voir entrer entièrement dans la voie qui mène à la perfection.

Tout paraissait donc aller pour le mieux quand on apprit, avec consternation, le prochain départ du fondateur pour un long voyage en Europe et en Terre sainte.

Cependant, par-delà les mers, monseigneur ne cessait de penser à ses filles spirituelles et entretenait avec elles une correspondance suivie. De Jérusalem, il leur écrit:

Mes chères Filles,

Ce matin, deuxième anniversaire mensuel de votre vie canonique, je vous ai nommées et recommandées à notre divin Sauveur, au Mont de l'Ascension, le suppliant de vous donner des pensées, des sentiments et des aspirations célestes. Vous êtes constamment dans ma pensée. Chaque matin, à la sainte Messe, et souvent durant le jour, en récitant le saint bréviaire, je répète les paroles du bon Maître à son Père céleste: • Père Saint, gardez celles que vous m'avez données. .

Une couvée qui n'a que deux mois cherche encore l'aile maternelle pour s'abriter ! Or, vous avez le Dieu de votre cœur et le Cour adorable de votre Dieu pour vous reposer, vous abriter, vous cacher. Goûtez-y la paix, trouvez-y la protection, cherchez-y une retraite où le monde ne puisse vous atteindre.

Dans une autre lettre, il écrit:

Aujourd'hui, 24 juin, vous comptez trois mois de vie religieuse canonique; de Montréal, de Jérusalem, de Rome, je vous écris les mêmes vœux de persévérance par une fidélité consciencieuse à la grâce qui vous sollicite, et par un redoublement de générosité dans le sacrifice. [...]

Et, plus loin:

J'ai visité à Rome, l'Institut normal Catholique de France. Ce sont des religieuses avec le costume des dames du monde. Elles occupent en France et en Italie une place d'honneur à la tête de l'enseignement et préparent des maîtresses d'écoles. Elles sont savantes et encore plus pieuses et plus humbles que savantes.

Votre situation près de l'Ecole normale, à Saint-Boniface, est un appel continuel à l'étude, à la formation normale de bonnes maîtresses, de vraies éducatrices.

Les mois passaient et monseigneur ne parlait pas de retour. Pourtant, le ciel s'assombrissait... Aux yeux de plusieurs, l'œuvre entreprise était inutile; on la disait même nuisible aux institutions 
déjà existantes auxquelles elle ravirait des sujets et des ressources. Selon toutes les prévisions humaines, l'œuvre était destinée à mourir, après avoir apporté à son fondateur déception sur déception.

Qui s'étonnera que les colombes du noviciat aient senti leurs ailes bien courtes pour s'élever, seules, au-dessus d'une atmosphère tout à fait défavorable à leur envol.

Monseigneur, faisant allusion aux difficultés de cette époque, écrira plus tard à l'une de ses filles: "Si la conduite étonnante de Dieu sur chacune de vous, au milieu d'épreuves terribles, qui auraient dû vous éloigner à jamais, n'est pas comme sa signature, son sceau posés sur les Oblates, je ne comprends plus rien aux œuvres de Dieu.»

C'était bien cette marque divine qui s'imprimait sur l'œuvre. Pendant que la main de Dieu travaillait à façonner et à unir les nouvelles novices dans la souffrance, le fondateur s'occupait, en Europe, de leur donner un signe de ralliement. Le 24 août, il leur écrit:

Aujourd'hui, cinquième anniversaire de votre vie de novice, je reçois de Monseigneur Legal, 0.M.I., le blason que je l'avais prié de tracer pour vous.

Toutes les lettres du fondateur ne respirent que la plus franche confiance en l'avenir. Ses filles avaient un nom, des armes, une devise: les Oblates du Sacré-Cœur et de Marie Immaculée sortaient, pour ainsi dire, de l'ambigu; leur voie se dessinait graduellement et leur offrait une perspective capable de tenter les ambitions les plus hautes. Hélas! les paroles si encourageantes de monseigneur ne devaient plus trouver un écho fidèle dans tous les cœurs.

Dans une semblable situation, on comprend facilement que l'annonce $\mathrm{du}$ prochain retour du fondateur fut, pour plusieurs, une grande joie.

Quelques jours après son arrivée, le calme se rétablit; les vides faits dans les rangs se comblaient par l'entrée au noviciat de quatre postulantes. Le 7 décembre avait lieu une seconde cérémonie qui ramenait à sept le nombre des novices.

L'année 1905 commença dans la quiétude que faisait naître la présence de Celui que les sœurs se plaisaient à considérer comme l'appui et le soutien de leur avancement dans la voie qu'elles avaient choisie.

Le travail était assez considérable pour employer tous les bras. Des religieuses et quelques jeunes filles, appelées à suivre les cours de l'École normale, trouvaient à la Maison-Chapelle le logement et la pension à proximité de leurs classes; la Maison gardait aussi des dames pensionnaires; les sœurs faisaient la typographie des Cloches de SaintBoniface, publication fondée en 1902. Mère Marie-Joseph-du-Sacré- 
Cour donnait des heures d'enseignement au personnel et de l'aide aux élèves de l'École normale.

Le fondateur voulut se réserver à lui-même d'expliquer à sa communauté les Constitutions déjà ébauchées en donnant des conférences sur la formation religieuse et sur le but de l'institut.

Mais monseigneur vit bientôt que la théorie et la pratique ne marcheraient pas de pair à moins que les novices ne soient guidées dans la pratique par une personne expérimentée. Il éprouva une agréable surprise en apprenant que, non seulement ses filles étaient prêtes à accepter sa proposition, mais qu'elles avaient elles-mêmes pensé à lui demander ce guide dans leur inexpérience.

La question n'était pas facile à résoudre. Monseigneur s'en aperçut bien vite après avoir renouvelé sa demande en vain auprès des supérieures de nos communautés-sœurs.

Mais la Providence, cette fois encore, veillait sur la jeune famille. A travers les événements regrettables qui se produisaient en France, elle préparait à la jeune congrégation le secours opportun.

Le fondateur adressa donc sa requête à la congrégation des Filles de la Croix de Saint-André, de La Puye, France, dont une maison venait d'être acceptée dans le diocèse. La réponse fut favorable. Pour reconnaître la bienveillance dont elles avaient été l'objet de la part de l'archevêque, les supérieures de cette communauté acquiescèrent à son désir en mettant à sa disposition une religieuse déjà exercée à la conduite des âmes. Le choix tomba sur la sœur Agnès-Émilie, supérieure à Winnipeg. Durant onze ans elle avait été maîtresse des novices en sa propre communauté.

Monseigneur lui explique dans une lettre ce qu'il attendait d'elle comme guide dans les exercices du noviciat et dans l'initiation aux vertus et à la pratique des vœux de religion. En voici deux paragraphes:

Vous êtes priée de bien vouloir étudier chaque sujet afin de pouvoir me dire, devant Dieu, ce que vous en pensez au point de vue de la vocation et de la profession religieuse.

...L'Institut qui commence est bien différent du vôtre; mais je désirerais beaucoup qu'il en eût d'abord l'esprit profondément religieux en tout ce qui regarde la sainte obéissance, à l'intérieur et à l'extérieur, puis l'amour de la pauvreté, l'esprit de charité fraternelle, et enfin, la bonne simplicité.

Cette lettre, impossible à résumer, montre bien où en était la congrégation à ce moment-là. A partir du 26 juin - et durant quatre mois - la sœur Agnès-Émilie, Fille de la Croix, fut maitresse de novices des Oblates. Ce geste charitable ouvre une ère de progrès et d'affermissement pour l'œuvre, si bien que monseigneur Langevin juge les cofondatrices suffisamment prêtes à remplir les obligations des vœux. Il fixa 
au 29 septembre la date de la première cérémonie de profession. Toute la petite famille vit dans cet événement une marque de la bénédiction du ciel.

Ĩonseigneur l'archevêque prếside la cêrémonie et monseigneur Z. Racicot, évêque auxiliaire de Montréal, donne le sermon. A l'issue de la cérémonie, monseigneur l'archevêque confirme les deux nouvelles professes dans les charges qu'elles occupaient déjà: Mère Saint-Viateur, âgée de 33 ans, supérieure, et Mère Marie-Joseph-du-Sacré-Cœur, assistante, en comptait 25.

L'année 1906 réalise le but particulier de la nouvelle congrégation: la fondation du couvent de Saint-Charles.

La ruche était-elle donc devenue trop étroite, ou les abeilles trop nombreuses? De toute façon, les sœurs ouvrent un couvent à SaintCharles. Le souci du bien des âmes est le premier mobile qui portait monseigneur à presser cette fondation.

1906 apporte aussi la bénédiction particulière de Sa Sainteté Pie X.

Entre temps, monseigneur Langevin écrivait de Rome:

Bénissez le bon Dieu de tout votre cœur. J'ai parlé de vous au Saint-Père, en toute simplicité, mais avec une certaine crainte. Or, le bon Pie X a approuvé votre petite Euvre en m'assurant qu'elle n'était pas du tout visée par son Motu Proprio sur les nouvelles congrégations qui surgissent en Europe. C'est pour moi un immense encouragement parce que c'est l'expression de la volonté de Dieu. Après avoir entendu le Vicaire de Jésus-Christ lui-même me dire d'aller de l'avant, je vais me mettre à l'œuvre avec une nouvelle ardeur pour assurer à notre jeune pays, le secours précieux d'un nouvel institut pour l'éducation chrétienne des enfants.

Le 10 mars 1909, la petite congrégation avait été reconnue comme institution civile sous le nom: "Les Missionnaires Oblates de SaintBoniface ", par un acte d'incorporation rédigé par le juge Prud'homme et présenté par monsieur Joseph Bernier, député de Saint-Boniface.

La communauté commençait donc à prendre sa place parmi ses devancières dans l'apostolat, mais il lui manquait encore le sceau de la stabilité que pouvait seul donner l'émission des vœux perpétuels.

Le 18 février 1911, Mères Saint-Viateur et Marie-Joseph-du-SacréCœur prononçaient leurs vœux perpétuels entre les mains du père fondateur. Mère Saint-Viateur avait comme marraine Mère Despins, supérieure-vicaire des Sœurs Grises de la Charité, de Saint-Boniface, et Mère Marie-Joseph-du-Sacré-Cœur, Mère Marie-Léonie, provinciale des Sœurs des Saints Noms de Jésus et de Marie, de Winnipeg. Étaient au chœur monseigneur Charlebois, o.m.i., et vingt-cinq prêtres.

La Mère Valérie Saint-Jean, supérieure des Filles de la Croix, occupait un siège réservé. Sa présence rappelait les services précieux 
que sa congrégation avait rendus à la nôtre. Dans la nef, les membres de sept communautés de femmes, les parents des religieuses et bon nombre d'amis.

Au soir de cette fête, dans l'intimité de la communauté, monseigneur rendait hommage aux cofondatrices. De Mère Saint-Viateur, il dit:

Dieu seul sait combien elle a dû se renoncer pour se donner à l'Euvre, alors que son cœur allait à une autre fondation commencée, et que tout semblait désespéré à la Maison-Chapelle, à la suite de plusieurs années d'essais infructueux. Son obéissance, bénie de Dieu, lui a fait accomplir, avec succès, une tâche colossale au point de vue humain. Et puis, le divin Maître lui a assigné, dès le début, en la personne de Mère Marie-Joseph-du-Saré-Cœur, une compagne merveilleusement apte à la seconder, en se donnant au travail intérieur et si délicat de la formation des novices. Un grand esprit de foi, une rare perspicacité et le talent de se gagner les cours, sans pourtant jamais sacrifier à la crainte de dire la vérité qui éclaire et guérit, ont fait d'elle une véritable maîtresse capable de préparer des sujets à la fois solides, éclairés et dévoués.

\section{Il ajoutait:}

Depuis le jour de l'érection canonique du noviciat, il y a eu, dans le silence, le travail pénible et la prière fervente de Nazareth, les angoisses de Gethsémani, et même souvent, le crucifiement du Calvaire. Les œuvres de Dieu qui commencent, vrai métal en fusion, mêlé de scories, doivent être jetées dans le creuset pour en sortir plus jeunes et plus vigoureuses.

La petite congrégation se développait normalement. Jusque-là, elle avait bénéficié largement de l'autorité et de la tendresse de son fondateur. Le jour allait venir où, laissée à elle-même, elle aurait à lutter, seule, pour son maintien et son développement.

Monseigneur Langevin souffrait depuis plusieurs années de la maladie qui devait l'emporter. Il était allé, chaque année, depuis quatre ans, demander à un climat plus doux le regain de forces dont il avait besoin pour soutenir la tâche qui devenait de plus en plus ardue. A l'automne de 1914, c'est vers le Texas qu'il se dirigeait avec l'espoir d'un micux sensible dans son état de santé.

Avant son départ, il remettait à Mère Saint-Viateur, supérieure générale, la dernière partie des Constitutions avec une lettre qui est restée mémorable. Il y écrivait entre autres choses:

Maintenant, Nous sentons le besoin de jeter un regard en arrière, jusqu'au jour où Nous vous avons donné, Nous-même, l'existence canonique, pour remercier Dieu, dans l'effusion de Notre cœur, le Cœur adorable de Jésus, de vous avoir bénies d'une façon si remarquable. Si votre œuvre n'avait pas été voulue de Dieu, elle aurait péri sous le coup des oppositions humaines, ou faute de sujcts dans un pays si nouveau. Mais le bon Maître a voulu que des prêtres zélés de la ville de Montréal et d'ailleurs viennent à Notre aide, en envoyant des sujets choisis qui Nous ont permis, non seulement de créer l'Euvre, mais de la développer.

Le vénéré fondateur y ajoutait des avis et des conseils qui sont restés dans les cœurs comme un testament, puisque la mort devait le 
surprendre loin de sa ville épiscopale, loin de ses diocésains, loin de ses filles de prédilection.

A Québec, où il était allé célébrer les noces d'or du vénérable cardinal Bégin, inunseigneur Langevin vil son élat s'aggraver d'une complication qui devait, en trois jours, avoir raison de sa vigoureuse constitution. Le mardi 15 juin 1915, il s'endormait dans la paix du Seigneur, à huit heures du matin, à l'Hôtel-Dieu de Montréal, à l'âge de 59 ans.

Cette mort fut un grand deuil pour le diocèse. Elle le fut davantage pour ses filles qui devenaient orphelines à onze ans... De toutes les leçons que leur avait données leur bien-aimé père, elles garderont, dans leur douleur, l'inébranlable confiance dans l'avenir, parce qu'elles se sentaient désormais Filles de Dieu et Filles de l'Église.

Dieu et l'Église ne manquèrent pas aux petites Oblates de SaintBoniface. Elles trouvèrent un guide et un protecteur en celui que Rome avait donné à monseigneur Langevin pour auxiliaire. Celui qu'elles pleuraient, avec tant de raison, revivait dans l'homme de son choix: monseigneur Arthur Béliveau. Et il en fut ainsi de chacun des chefs spirituels qui se succédèrent sur le siège archiépiscopal de Saint-Boniface.

Pour finir, quelques dates et statistiques:

1923 - Achat de l'École normale française, rue Masson

1928 - Construction à l'épreuve du feu du Jardin Langevin

1933 - Monseigneur Émile Yelle, archevêque-coadjuteur de Saint-Boniface, constate le besoin d'une organisation pour la jeunesse féminine; monsieur l'abbé Léo Blais, alors vicaire à la Cathédrale, en est l'aumônier; le noyau de jeunes filles, récollectantes de la Maison-Chapelle, devient la première cellule des mouvements spécialisés d'Action catholique de la Jeunesse féminine du diocèse; les Sœurs Oblates mettent des locaux à la disposition de la jeunesse féminine dans l'ancienne École normale; on y voit apparaître la Jeunesse ouvrière catholique féminine (J.0.C.F.), les guides â̂nées, les catéchistes pour l'enseignement oral dans la paroisse et l'enseignement par correspondance dans le diocèse; à ces mouvements se rattachent: le Bureau de placement pour jeunes filles, la Bibliothèque paroissiale, la Librairie catholique, les Colonies de vacances, l'École familiale, le Foyer pour étudiantes et jeunes ouvrières, et autres œuvres semblables

1942 - Les Sours Oblates font l'achat d'une propriété, rue Langevin, autrefois rue du Collège, pour l'EEuvre des retraites fermées 
1948 - Approbation pontificale de nos Constitutions

1948 - Construction d'une infirmerie reliée à la vieille maison

1957 - Construction de la maison mère actuelle

1957 - Décès de Mère Saint-Viateur; elle était dans sa $87^{e}$ année

1958 - Mère Marie-Joseph-du-Sacré-Cœur entre dans la paix du Seigneur à l'âge de 77 ans

La congrégation compte actuellement 280 religieuses qui œuvrent en HUIT diocèses répartis dans CINQ provinces dont:

22 centres d'apostolat au Manitoba

2 en Ontario

5 en Saskatchewan

7 en Alberta

1 à Québec

A la maison mère, des sœurs s'occupent du catéchisme par correspondance, de l'enseignement religieux des sourds-muets à Tuxedo, et au Centre Jean-Bosco, pour les Indiens et les Métis, d'autres collaborent au service social de la paroisse, et aux travaux de la Croix-Rouge. SEIZE (16) sont à l'étude à plein temps.

Cependant, l'accroissement n'est pas l'essentiel pour un corps religieux. Ce qui compte, c'est de jouer son rôle parmi les œuvres de Dieu selon l'esprit qui lui a été donné.

Puissions-nous, à l'exemple de notre bien-aimé fondateur, garder le dépôt!

Sœur Cécile d'Amours, m.o., Maison généralice des Missionnaires Oblates, Saint-Boniface, Manitoba. 